\title{
Global magnetic field cycle evolution and prominence eruptions
}

\author{
Irina A. Bilenko \\ Moscow M. V. Lomonosov State Univercity, Sternberg Astronomiczl Institute, Moscow, Russia \\ email: bilenko@sai.msu.ru
}

\begin{abstract}
A comparison of changes in the structure of the global solar magnetic field and that in the prominence parameters, in solar cycles 21-23, are presented. It is proposed that the observed global magnetic field structure changes and periodicities in the mean solar magnetic field are the result of the excitation of large-scale Rossby waves. The changes in the prominence parameters are assumed to be the result of the global magnetic field structure changes, which may be triggered or modulated quasi-periodically by large-scale Rossby waves.
\end{abstract}

Keywords. magnetic fields, prominences, oscillations.

\section{Introduction}

Prominences are large cool plasma condensations in the low solar corona, typically 2 orders of magnitude denser and cooler than the million-degree coronal plasma. They appear only above long-lived magnetic channels in the chromosphere, trapped by the underlying magnetic fields (Tandberg-Hanssen 1995; Martin 1998). Although solar filaments are observed at all latitudes on the Sun, they always form above polarity inversion lines, which divide regions of positive and negative magnetic fields. Solar prominences are associated with a wide variety of solar activity phenomena. A close relationship between prominence eruptions and coronal mass ejections (CMEs), and flares were established (Munro et al. 1979; Hori \& Culhane 2002; Gopalswamy et al. 2003). Prominences were one of the first activity phenomena associated with mass ejections from the Sun (Tandberg-Hanssen 1995). Prominences/filaments are also known to be the good traces of the evolutionary changes of the global solar magnetic field during solar cycles (Hyder 1965; Makarov \& Sivaraman 1989).

The aim of this paper is to investigate the dependence of prominence parameters on global magnetic field structure (GMFS) cycle evolution.

\section{Data}

The mean magnetic field data and source surface synoptic maps from the Wilcox Solar Observatory were used. The coronal magnetic field is calculated from photospheric fields with a potential field model with the source surface location at 2.5 solar radii (Altschuler \& Newkirk 1969; Schatten, Wilcox \& Ness 1969; Hoeksema, Wilcox \& Scherrer 1983).

Data on prominence parameters were taken from the Kislovodsk Mountain Astronomical Station of the Pulkovo Observatory. The daily observations on solar prominences and their parameters, such as area, height, and length were carried out on the station in line $H \alpha(\lambda=6563 \AA)$ since 1957 (Gnevyshev et al. 1963; Guseva et al. 2006).

\section{Results}

The longitudinal distribution of positive-polarity and negative-polarity magnetic fields, resulting from the PFSS extrapolation at 2.5 solar radii, is displayed in Fig. 1a. In Fig. 1b the longitudinal diagram of the mean solar magnetic field is presented. The brightness at a certain point is proportional to the magnetic field strength, averaged over latitude, 


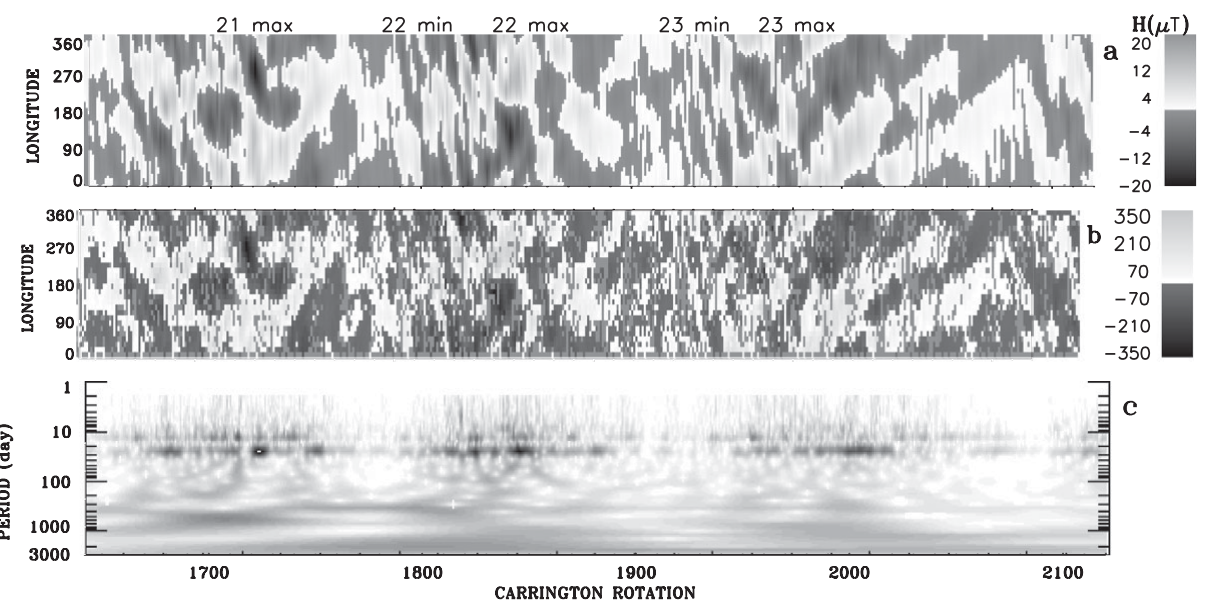

Figure 1. The longitudinal diagrams of (a) coronal magnetic field; (b) the mean solar magnetic field. Light denotes positive-polarity magnetic fields and dark - negative-polarity ones. (c) Wavelet power spectra of the mean solar magnetic field.

for each Carrington rotation (CR). The coronal magnetic field is anchored in the subphotospheric magnetic fields and is forced to evolve in accordance with the changing photospheric magnetic fields. Magnetic fields form a multi-scale GMFS, depending on the phase of a cycle. The largest structures, with the life-time $\sim 1$ to 5 years, were observed during the maxima and declining phases. There were three fast $(\sim 1$ to 3 solar rotations) redistributions of the GMFS, covering a considerable part of the solar surface, during the maxima and declining phases. Magnetic structures during the minima and rising phases were smaller compared to those of the maxima and declining phases.

Gilman (1969) proposed, that observed solar magnetic fields can be the result of Rossby waves generated in the Sun's convection zone. In Tikhomolov $(1995,1996)$ the Rossby vortices, excited within a thin layer beneath the convection zone, were considered to explain the observed GMFS. The Rossby vortices are the result of heating from the solar interior and the deformation of the convection zone lower boundary. According to Zaqarashvili et al. (2010a, 2010b), the periodicity of 155-160 days and $\sim 2$ years, observed in different solar activity indices, can be connected to the dynamics of magnetic Rossby waves in the solar tachocline, since in the layer they are unstable due to the joint effect of the toroidal magnetic field strength and latitudinal differential rotation.

The wavelet power spectra of the mean solar magnetic field is shown in Fig. 1c. It is proposed, that the GMFS in Fig. 1a and 1b is a consequence of the excitation of Rossby waves of different periods during different solar cycle phases. Noteworthy is the fact that the wave period do not remains constant, but undergoes abrupt changes. There were several "switches" in wave periods during each maxima of cycles $21-23$ : from $300 \mathrm{~d}-400 \mathrm{~d}$ to $40 \mathrm{~d}-$ $50 \mathrm{~d}$ and then to $300 \mathrm{~d}-400 \mathrm{~d}$ again. The intensity of the waves was different in different cycles. These changes in wave periods are reflected in the reorganizations of the GMFS. So, the GMFS could be a consequence of the exitation of Rossby waves of different periods.

Fig. 2 shows the evolution of prominence parameters such as (a) daily counts of prominence events $(\mathrm{N}),(\mathrm{b})$ prominence heights, (c) length, and (d) areas. Dots represent data for each prominence, thin solid lines represent CR averaged data (the scales are shown on the right y-axis). The latitudinal distributions of prominences depending on their area are shown in Fig. 2(e-h). It is known that the parameters of prominences varies with solar cycles. From Fig. 2(a-d) it is seen that in addition to the general cycle variation in the prominence parameters, the local changes are also observed. The comparison of Fig. 1 

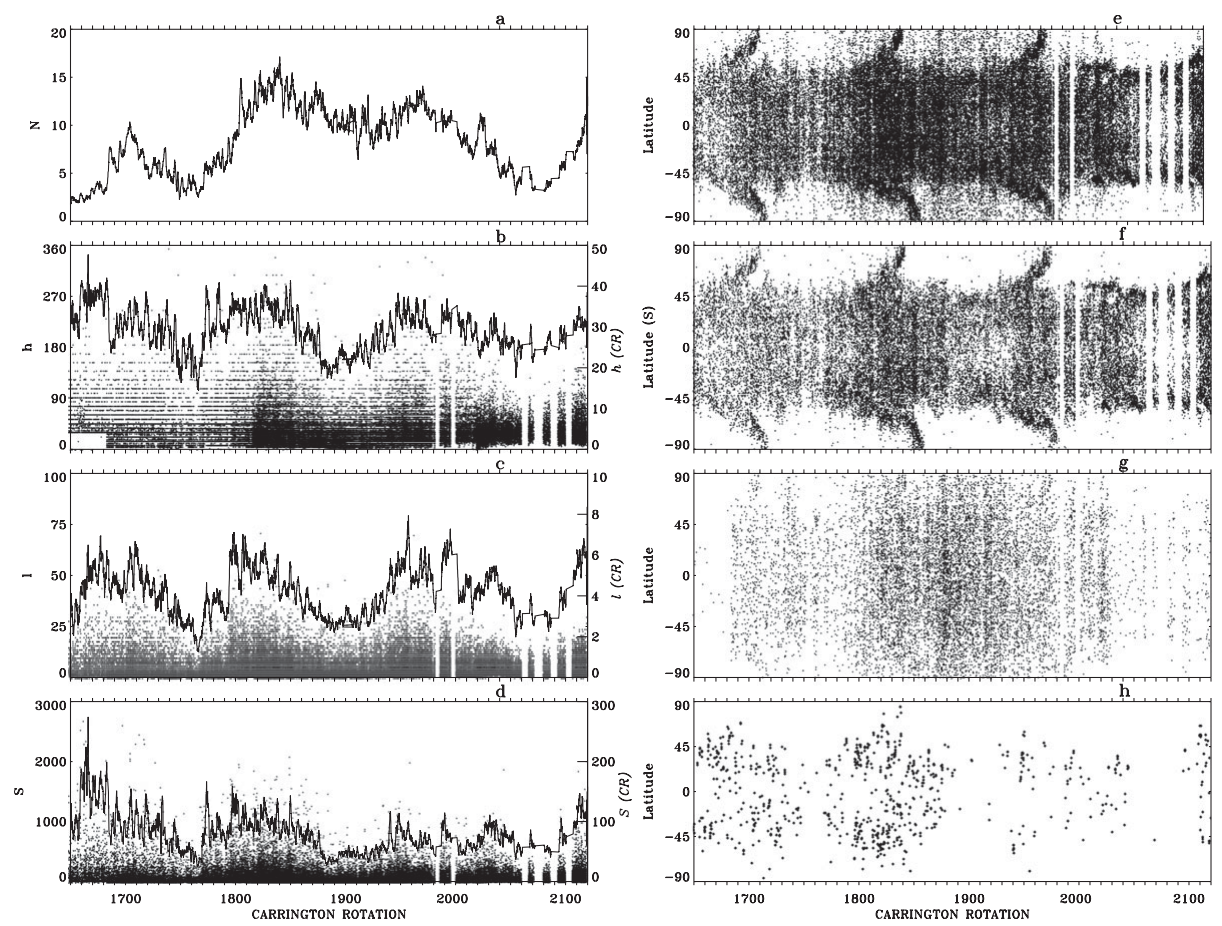

Figure 2. Prominence parameters.

and 2 shows that the number of prominences was higher in times of the GMFS reorganization. Prominences had, on average, lower height, length, and area. Thus, changes in the GMFS determine the number and parameters of prominences. The reorganization of the GMFS result in the growth of weak CME events (Bilenko 2012), that can be associated with some prominence eruptions. This may be caused by the changes in Rossby wave regime generation and a new magnetic flux emergence, resulting in the GMFS reorganization, which in turn leads to an increase in the number of prominence eruptions and associated CMEs. The coronal magnetic field strength follows the GMFS evolution and undergoes abrupt changes, reflecting changes in activity within the large-scale magnetic patterns. The field strength decreases during the times of the reorganization of the GMFS. The strength of magnetic fields surrounding prominences play an important role in the filaments/prominences evolution (Svetska 1986). Decrease of magnetic field strength could be responsible for some prominence/filament eruption (Schmieder et al. 2008). The prominence eruptions can be also the result of the removal of the restraining coronal magnetic field. When the coronal magnetic structure is destroyed and the field diminishes, the force, which prevent a filament/prominence from eruption, decreases and a prominence can erupt. During the reorganization of the structure of the magnetic field in the solar corona the conditions are not stable. Therefore, large, long, prominenves/filamens do not have time to form, and, hence, they are smaller in size and length. Moreover, since external magnetic field, preventing an eruption, decreases, the critical height (Filippov et al. 2006) for prominences to erupt will be lower.

It is well known that the latitude distribution of prominences and their activity changes during solar cycles (Gnevyshev \& Makarov 1985; Lorenc, Pastorek, \& Rybanský 2003; Shimojo et al. 2006). The latitudinal interrelation of prominence eruptions and CMEs depends on a solar-cycle phase (Gopalswamy et al. 2012). From Fig. 2(e-h) it is seen that the behaviour of prominences with different parameters is different. Prominences with an 
area more that 700 (height $>100 "$, length $>25^{\circ}$ ) change their position in latitude during cycles (Fig. 2h), whereas prominences with an area less than 15 (7”, $2^{\circ}$ ) are distributed uniformly (Fig. $2 \mathrm{~g}$ ). For these prominences, the concentration to the moments of the GMFS reorganization is observed. The drift of prominence location to the north and the south poles is most pronounced for that in size from 40 to 600 (80"-100", $\left.10^{\circ}-15^{\circ}\right)$ (Fig. 2f). The drift occurred when the large-scale GMFS formed.

In summary, from the above, we can conclude that the changes in the prominence parameters, during solar cycles, could be the result of GMFS changes, which may be triggered or modulated quasi-periodically by large-scale Rossby waves.

\section{Acknowledgements}

I would like to thank the Local Organizing Committee and IAU for the financial support and the chance to present at the conference. Wilcox Solar Observatory data used in this study was obtained via the web site http : //wso.stanford.eduat2011 : 03 : 22_05 : 18 : 09 PDT courtesy of J.T. Hoeksema. The Wilcox Solar Observatory is currently supported by NASA. Kislovodsk Mountain Astronomical Station of the Pulkovo Observatory data was obtained via the web site //www.solarstation.ru.

\section{References}

Altschuler, M. D. \& Newkirk, G. 1969, Solar Phys., 9, 131

Bilenko, I. A. 2012, Geomagnetism and Aeronomy, 52, 1005

Filippov, B. P., Zagnetko, A. M., Ajabshirizadeh, A., \& Den, O. G. 2006, Sol. Syst. Res., 40, 319

Gilman, P. A. 1969, Solar Phys., 8, 316

Gnevyshev, M. N. 1963, AZh, 40, 401

Gnevyshev, M. N. \& Makarov, V. I. 1985, Solar Phys., 95, 189

Gopalswamy, N., Shimojo, M., Lu, W., Yashiro, S., Shibasaki, K., \& Howard, R. A. 2003, ApJ, 586,562

Gopalswamy, N., Yashiro, S., Mäkelä, P., Michalek, G., Shibasaki, K., \& Hathaway, D. H. 2012, ApJ, 750, L42

Guseva, S. A., Kim,G.-D. \& Tlatov, A. G. 2007, in proc. "Multiwavelength investigations of the Sun and the problems in solar activity" SAO RAN, publ. Sankt-Peterburg, p. 269

Hoeksema, J. T., Wilcox, J. M., \& Scherrer, P. H. 1983, JGR, 88, 9910

Hori, K. \& Culhane, J. L. 2002, A\&A, 382, 666

Hyder, C. L. 1965, ApJ, 141, 272

Lorenc, M., Pastorek, L., \& Rybanský, M. 2003, in proc. ISCS 2003 Symposium "Solar Varuability as an Input to the Earth's Environment", Tatranská Lomnica, Slovakia, p. 129

Makarov, V. I. \& Sivaraman, K. R. 1989, Solar Phys., 123, 367

Martin, S. F. 1998, Solar Phys., 182, 107

Munro, R. H., Gosling, J. T., Hildner, E., MacQueen, R. M., Poland, A. I., \& Ross, C. L. 1979, Solar Phys., 61, 201

Schatten, K. N., Wilcox, J. M., \& Ness, N. F. 1969, Solar Phys., 6, 442

Schmieder, B., Bommier, V., Kitai, R., Matsumoto, T., Ishii, T. T., Hagino, M., Li, H., \& Golub, L. 2008, Solar Phys., 247, 321

Shimojo, M., Yokoyama, T., Asai, A., Nakajima, H., \& Shibasaki, K. 2006, PASJ, 58, 85

Svetska, Z. 1986, The Lower Atmosphere of Solar Flares, NSO/Sac Peak Pub., 332.

Tandberg-Hanssen, E. 1995 The Nature of Solar Prominences, Kluwer Acad., Norwell, Mass.

Tikhomolov, E. 1995, Solar Phys., 156, 205

Tikhomolov, E. \& Mordvinov, V. I. 1996, ApJ, 472, 389

Zaqarashvili, T. V., Carbonell, M., Oliver, R., \& Ballester, J. L. 2010a, ApJ, 709, 749

Zaqarashvili, T. V., Carbonell, M., Oliver, R., \& Ballester, J. L. 2010b, Ap. Lett., 724, L95 